\title{
Phänomen ohne Wirklichkeit
}

Ein Kommentar zu H. MEYERS „Der Hochleistungssport - Ein Phänomen des Showbusiness“

Die Kritik an H. MEYERS Aufsatz bezweckt im wesentlichen, auf die Folgen undialektischer und ahistorischer Schritte bei der Analyse dialektisch konstituierter sozialer Beziehungszusammenhänge aufmerksam zu machen: transparent bleibt am konkreten Beispiel des Verhältnisses von Hochleistungssport und Gesellschaft ein idealistischer Dualismus von Individuum und Gesellschaft, dessen Erscheinungsform sich in einer subjektivistischen Auffassung gesellschaftlich handelnder Individuen manifestiert. Darüber hinaus erweist sich durch den vieler historischer und gesellschaftlicher Bedingungen entkleideten Übertrag polit-ökonomischer Kategorien aus der Sphäre konkreter gesellschaftlicher Produktion auf den Bereich des Hochleistungssports, wie sich letzterer dennoch, trotz gegenteiliger Beteuerungen des Autors, als ,,gesellschaftsenthoben" präsentiert.

1) Die von H. MEYER gewählte Ebene, mit der Kritik gerade am Selbstverständnis des Sports - wie es sich in der Freiraum-Ideologie manifestiert - anzusetzen, setzt für den Autor sachlogisch determinierte Begrenzungen. Gemäß dieser perspektivistischen Verengung bleibt seine Kritik an Erscheinungsformen behaftete Detailkritik. Konsequenz einer solchen Verfahrensfolge ist es, Sport und sein mögliches Selbstverständnis nicht in soziologischen Kategorien begreifen zu können, die dialektische, historische, soziale und prozessuale Momente widerspiegeln; als notwendige Folgen kann lediglich eine im Kontrast zum sportlichen Selbstbild stehende Bestimmung des Hochleistungssports als von Leistungsausrichtung diktiert erscheinen und somit keine grundlegende, mit historischen, sozialen und bei alledem kritischen Kategorien gefuihrte Auseinandersetzung innerhalb des Problemzusammenhangs möglichen Selbstverständnisses des Sports und der sportlichen Realität erreicht werden (PROKOP 1971: 22ff.). Wenn Exterritorialität und Pazifizierung als angenommene funktionale Merkmale des Selbstbildes im Sport einmal aus dem geschichtlichen und gesellschaftlichen Zusammenhang herausgenommen werden, erweitern die im Verlauf des Aufsatzes ausgearbeiteten Momente wie etwa Leistungsausrichtung, organisierter Sportbetrieb, Phänomene des Showbusiness etc. eine Skala von Aspekten im wesentlichen auf quantitativer Ebene unter Ausklammerung gesellschaftlicher Aspekte. So wie MEYER aber die Freiraum-Ideologie auf der nur wenig abstrakten ${ }^{1}$ Ebene und bar der Untersuchung der Ursprungsbedingungen darstellt, so erscheinen auch die Aspekte der Leistungsdominanz und des Warencharakters sportlicher Handlungen als geschichtlos und statisch angegeben. Sie stehen somit im Gegensatz zum wirklichen historisch-gesellschaftlichen Entwicklungsprozeß und sind somit für soziologische Erkenntnis irrelevant. Die wenig prinzipiell gefuihrte Analyse führt dazu, weder Ideologien überhaupt noch die Ideologie des Freiraums im besonderen als wichtigen Bestandteil des historisch-gesellschaftlichen Entwicklungszusammenhangs bestimmen zu können. Das Ergebnis, nämlich den Aspekt der Freiraum-Ideologie durch den Aspekt der Leistungsausgerichtetheit zu kontrastieren, macht es dem Autor darum immer noch möglich, dem Sport wenigstens ,die Freiraumexistenz seiner Gründerjahre" (MEYER 1973: 62) zuschreiben zu können. Darüber hinaus wird die Separation des Sports weiter dadurch betrieben, daß dem Hochleistungssport eine ,Eigenlogik“ zugestanden wird. Diese Perspektive verbaut endgiiltig den Weg, das dialektische Verhältnis von Sport und Gesellschaft auf dem Hintergrunde einer gesellschaftlichen Totalität, die ,kein Eigenleben oberhalb des von ihr Zusammengefaßten, aus dem sie selbst besteht" (ADORNO 1972: 127), führt, mit den adäquaten soziologischen Kategorien zu erfassen. Ins Blickfeld gerät lediglich eine diffuse Vorstellung einer „Verschränkung von Sport und Politik" (MEYER 1973: 61) etc. als auch das Bemühen um die Erkenntnis, ,wie sehr die politischen Kräfte neben der Wirtschaft um die Popularität des Sports wissen und sie in ihrem Sinne zu nutzen suchen“" (MEYER 1973: 61).

Auf dem Hintergrunde der gesellschaftlichen Dialektik von Teil und Ganzem, wo ein gesellschaftliches Element nicht verstanden werden kann ohne Erkenntnis des nur in Entwicklung

1 In der Bedeutung: die Ebene der Abstraktion allein erlaubt die Reproduktion konkreter Erscheinungsformen der gesellschaftlichen Realität im Rahmen des soziologischen Erkenntnisprozesses. 
begreifbaren Ganzen, welches an der Prozessualität der einzelnen sozialen Erscheinungen sein Wesen widerspiegelt, wo die gesellschaftliche Totalität, wie ADORNO sagte, ,sich durch ihre einzelnen Momente hindurch ... produziert und reproduziert" (ADORNO 1972: 127), wie auch in der Sichtweise einer historisch und gesellschaftlich begriffenen Dialektik von Individuum und Gesellschaft, die als eine wesentliche Voraussetzung den praktisch tätigen Menschen als das Subjekt der Geschichte hat und die die Existenz der gesellschaftlichen Totalität in der praktischen und erkennenden Tätigkeit der Individuen, die die Gesellschaft ausmachen, sieht, zeigt sich der von H. MEYER durchgängig vorgenommene Dualismus von Individuum und Gesellschaft. Den Aspekten der Exterritorialität, des pazifizierenden Geschehens im Sport usw. und dem durch totale Leistungsausrichtung skizzierten Hochleistungssport fehlt ganz einfach das tätige Subjekt, das Individuum, das erst im historischgesellschaftlichen Kontext auf dem Wege seiner gesellschaftlichen Arbeit in seinen prinzipiellen Abhängigkeiten, Interessen etc. freigelegt werden müßte.

Auf der hier eingenommenen Ebene von Verschränkungen und wechselseitigem Profitieren erscheint das dialektische Zusammenhängen aller sozialen Bereiche, welches konkret die Form motivierter politischer, institutionalisierter $\mathrm{Pla}$ nung und Organisation sportlicher und sportlichbürokratischer Handlungsabläufe annehmen kann, nicht mehr als Bestandteil gesellschaftlicher Praxis sondern eher als Produkt subjektiver Willkür und Einzelgeschicks. Ihr adäquater Ausdruck sind die Sprachfetischismen wie ,das ideologische Selbstbild des Sports“ (MEYER 1973: 59), beruhend ,auf dem opportunistischen Geschick seiner Führung und speziell des Internationalen Olympischen Komitees, ihre Neutralität faktisch zu verwirklichen als eine mehr oder weniger offene Koalition mit den Institutionen real-politischer Effizienz" (MEYER 1973: 61), so geschehen mit dem Beispiel „Baron DE COUBERTINS Cleverness" (MEYER 1973: 62). Nach MEYER,,sucht der Sport von der Präsenz prominenter Politiker zu profitieren und gestattet diesen auch, sich sportfreundlich und damit dem Wähler durchaus sympathisch zu demonstrieren“ (MEYER 1973: 61). Analysen dieser Art sind m.E. eher dazu geeignet, viele Fragen prinzipieller Natur nicht aufzuwerfen. Bei der Annahme o.g. individualisierter Ak- tivitäten muß gesehen werden, daß die Komplexität sozialer Handlungsabläufe nicht aus den empirisch wahrnehmbaren Aktivitäten selbst und ihren Zielsetzungen erklärbar ist, sondern als Produkt sozial-historischer Verhältnisse und damit in enger Verbindung zu diesen angesehen werden muß.

2) Die Absicht des Autors besteht u.a. darin, den Hochleistungssport nicht zwischen Spiel und Arbeit anzusiedeln ${ }^{2}$, sondern als eine Art des Showbusiness auf eigener sachlogischer Ebene zu begreifen. Bei der Betrachtung der angewendeten soziologischen Kategorien ,Warenproduktion einerseits und Konsumtion andererseits' fällt nun auf, daß sie, um in der weiteren Analyse verwertbar zu bleiben, in ihrer Bedeutung unter den Begriff des Showbusiness subsumiert wurden und damit zugleich aus ihrem ursprünglichen kategorialen Kontext herausgelöst worden sind. Bleibt einmal vom warenanalytischen Ansatz abstrahiert, der von B. RIGAUER schon 1969 in die soziologische Theorie des Sports eingebracht worden ist (RIGAUER 1969: $58 \mathrm{ff}$.), so ist zum Verständnis der Kategorien ,Ware‘, ,Warenproduktion“ und ,Konsumtion“, wie sie in der MARXschen Kritik der politischen Ökonomie zu finden sind, anzumerken, daß sie dort zumindest nicht in isolierter Weise gebraucht sind. Zur Kennzeichnung einer konkreten gesellschaftlichen Epoche und ihrer Art und Weise und Form der Produktion sind die o.g. Kategorien im Kontext der MARXschen Analysen nicht ohne ihren historisch-materialistischen Gehalt zu begreifen. MEYER überträgt ungerechtfertigt diese Kategorien und problematisiert nicht einmal den Begriff der ,Produktion sportlicher Darstellungen“. Der Verzicht auf die Kategorie gesellschaftlicher Arbeit in historisch-sozialer Konkretion beläßt die Analyse des Autors günstigstenfalls im Bereich der Konsumtion. In Anbetracht der undialektischen und geschichtslosen methodologischen Grundlagen dieses Aufsatzes, der ohne die wesentlichen, den Hochleistungssport konstituierenden Bedingungen und determinierenden Faktoren im konkreten gesellschaftlichen Kontext auskommt und der bei Ausklammerung der konkreten Form der Produktion und Repro-

2 MEYERS Kritik an LÜSCHEN ist in diesem Punkte nur möglich durch die beliebige Verwendung der Begriffe Sport und/oder Hochleistungssport. Vgl. MEYER 1973: $67 \mathrm{f}$. 
duktion des gesellschaftlichen Lebens somit diese Gesellschaft auch nicht benennen kann, kann der Autor wenig präzise das Verhältnis von Wirkung und Gegenwirkung im Hinblick auf seine Schlußfolgerung bestimmen. MEYERS Resümee: Das Konzept der Förderung des Breitensports erhalte reale Chance, weil sich der Hochleistungssport in seiner Entwicklung selbst von der statistischen Durchschnittsperson in die Isolation begebe. ,Eine solche Entfernung könnte dem Breitensport förderlich werden, wenn die Verbindung von Hochleistungssporterfolgen und nationalem Prestige sich in internationalen Profigruppen verlieren würde" (MEYER 1973: 77).

Dies alles könnte geschehen, wenn die herausgearbeiteten Aspekte von wesentlicher Bedeutung in der sozialen Wirklichkeit wären.

\section{Konstatierbare Phänomene Antwort auf HEINER ZIESMER}

Es mag sein, daß H. ZIESMER durch meinen Aufsatz ,,Der Hochleistungssport - ein Phänomen des Showbusiness" veranlaßt wurde, seine Gedanken niederzuschreiben. Das halte ich für eine psychologische Frage. Eine Erwiderung auf den genannten Aufsatz zur Soziologie des Sports stellen ZIESMERS Ausführungen jedenfalls nicht dar. Sie beschäftigen sich mit anderen Gegenständen, nicht mit den von mir in durchaus falsifizierbaren Aussagen thematisierten.

Das Kriterium empirischer Konstatierbarkeit der Phänomene signalisiert grundsätzliche Differenzen. Die Aussagen, die ich, ausgehend von der Kontrastierung verschiedener Akzente im Selbstbild des Sports mit Beobachtungen bei den Olympischen Spielen in München, über den Hochleistungssport machte, bezogen sich auf überprüfbare Gegebenheiten. In diesem Sinne intendierten sie eine raum-zeitliche Wirklichkeit, die intersubjektiv erfahrbar - und freilich in verschiedenartigen Theoriegefügen interpretierbar ist.

Der Bezug auf konstatierbare Phänomene fehlt in ZIESMERS Ausführungen. Bezeichnenderweise artikulieren sie zu den konkreten Beobachtungen und Interpretationen in meinem Aufsatz keinen greifbaren Widerspruch.

\section{Literatur}

ADORNO, T. W. 1972: Zur Logik der Sozialwissenschaften. In: T. W. Adorno u.a.: Der Positivismusstreit in der deutschen Soziologie. Neuwied und Berlin: Luchterhand.

MEYER, H., 1973: Der Hochleistungssport - Ein Phänomen des Showbusiness. Zeitschrift für Soziologie 2, 59-78.

PROKOP, U., 1971: Soziologie der Olympischen Spiele. Sport und Kapitalismus. München: Hanser.

RIGAUER, B., 1969: Sport und Arbeit. Frankfurt: Suhrkamp.

HEINER ZIESMER, stud. päd. 404 Neuss, Kölner Straße 73

Hält man sich an die gängigen Usancen - sie machen Kommunikation möglich! - für das Verständnis des Begriffs ,Wirklichkeit“, so dürfte kein Zweifel darüber bestehen, wer ein „Phänomen ohne Wirklichkeit" beschreibt. Dem mit psychologischen Gedanken und psychischen Mechanismen vertrauten Leser wird es aufschlußreich sein, daß ZIESMER seinen Ausführungen den Titel „Phänomen ohne Wirklichkeit" gab. Dieser Maßnahme würde der Charakter der suspekten Selbsteinschätzung und Projektion nur dann genommen, wenn ZIESMER - was er nicht tat - sein Verständnis des Begriffs Wirklichkeit ausdrücklich abweichend vom üblichen Inhalt definiert oder umschrieben hätte.

Der Begriff Sport bleibt in ZIESMERS Ausführungen ersetzbar durch ziemlich beliebig auszuwählende gesellschaftliche Partialphänomene. Dies bedeutet mit anderen Worten: dort wird generell gehandelt über die Interdependenz von gesellschaftlichen Teilphänomenen und gesellschaftlicher Totalität, zum Teil leider nur mit dem Referat sprachlich drapierter Banalitäten. Die streckenweise obskur-verschlungenen Wendungen erinnern an mißverstandenen Hegelianismus zweiter Hand, auch an Bekenntnisse höriger Adepten kultisch verehrter Meister; sie muten an wie die Mantras der indischen Religions- 